\title{
Demonstration of plant fluorescence by imaging technique and Intelligent FluoroSensor
}

Sándor Lenk, Patrik Gádoros, László Kocsányi, Attila Barócsi

Sándor Lenk, Patrik Gádoros, László Kocsányi, Attila Barócsi, "Demonstration of plant fluorescence by imaging technique and Intelligent FluoroSensor," Proc. SPIE 9793, Education and Training in Optics and Photonics: ETOP 2015, 97931 (8 October 2015); doi: 10.1117/12.2223179

Event: Education and Training in Optics and Photonics: ETOP 2015, 2015, Bordeaux, France 


\title{
Demonstration of plant fluorescence by imaging technique and Intelligent FluoroSensor

\author{
Sándor Lenk*, Patrik Gádoros, László Kocsányi, Attila Barócsi \\ Department of Atomic Physics, Budapest University of Technology and Economics, \\ 1111 Budafoki út 8, Budapest, Hungary
}

\begin{abstract}
Photosynthesis is a process that converts carbon-dioxide into organic compounds, especially into sugars, using the energy of sunlight. The absorbed light energy is used mainly for photosynthesis initiated at the reaction centers of chlorophyll-protein complexes, but part of it is lost as heat and chlorophyll fluorescence. Therefore, the measurement of the latter can be used to estimate the photosynthetic activity. The basic method, when illuminating intact leaves with strong light after a dark adaptation of at least 20 minutes resulting in a transient change of fluorescence emission of the fluorophore chlorophyll-a called 'Kautsky effect', is demonstrated by an imaging setup. The experimental kit includes a high radiant blue LED and a CCD camera (or a human eye) equipped with a red transmittance filter to detect the changing fluorescence radiation. However, for the measurement of several fluorescence parameters, describing the plant physiological processes in detail, the variation of several excitation light sources and an adequate detection method are needed. Several fluorescence induction protocols (e.g. traditional Kautsky, pulse amplitude modulated and excitation kinetic), are realized in the Intelligent FluoroSensor instrument. Using it, students are able to measure different plant fluorescence induction curves, quantitatively determine characteristic parameters and qualitatively interpret the measured signals.
\end{abstract}

Keywords: chlorophyll-a, fluorescence induction kinetics, laboratory practice, photosynthetic activity, pulse amplitude modulation, excitation kinetics

\section{INTRODUCTION}

In 1931, H. Kautsky and A. Hirsch explored that the fluorescence emitted by the dark adapted plants is changing with the irradiation and its temporal variation is related to the photosynthesis ${ }^{1}$. Since it is an active effect which is influenced by several physiological and environmental interactions, it allows a deeper insight into the chemical and biological processes of the examined tissue compared to other, often claimed as passive, optical techniques (e.g. reflection or transmission). The chlorophyll (Chl) fluorescence of leaves consists of two maxima in the red (near 685-690 nm), and far-red region (near 730-740 nm). The intensity and shape of the Chl fluorescence emission spectrum of leaves at room temperature are dependent on the concentration of the fluorophore Chl- $a$, on the leaf structure, on the photosynthetic activity, and on the leaf's optical properties ${ }^{2}$. However, the fluorescence induction kinetics (or transient) is caused by the change of the fluorescence yield with the onset of various processes of photosynthesis during the dark-to-light transfer ${ }^{3}$. Furthermore, a re-absorption is caused by the overlapping of the short wavelength range of the Chl fluorescence emission spectrum with the long-wavelength range of the Chl absorption spectrum, which can be used to estimate the active Chl content ${ }^{2}$.

The aim of the paper is to present practical experiments, which introduce students into this biophysical, plant physiological research field. The laboratory practice is starting with a demonstration of the 'Kautsky kinetic' using an imaging setup. Then it continuous with a fluorescence kinetic measurement using a compact, intelligent fluorosensor (IFS), developed for basic and applied research on this field.

\section{DEMONSTRATION WITH IMAGING EQUIPMENT}

Intact leaves, illuminated with strong light after a dark adaptation of at least $20 \mathrm{~min}$, exhibit a transient change of fluorescence emission of the fluorophore Chl- $a$ which is called 'Kautsky effect' ${ }^{1}$. Time resolved investigation of the Chl$a$ fluorescence is widely used in basic and applied photosynthesis research ${ }^{3}$. For inducing photosynthetic activity, an actinic light is used which should be saturating, when measuring with continuous mode detection, or which should not be saturating when measuring with synchronous detection based on pulse amplitude modulation (PAM) ${ }^{3}$. Recently, PAM

*lenk@eik.bme.hu; phone 36 1 463-4201; fax 361 463-4201; fat.bme.hu

Education and Training in Optics and Photonics: ETOP 2015, edited by Eric Cormier, Laurent Sarger Proc. of SPIE Vol. 9793, 97931T · C 2015 SPIE, IEEE, OSA, ICO · doi: 10.1117/12.2223179 
has become the most commonly used fluorescence induction technique for plant physiological studies and precision agriculture.

However, we think that students need to see - likely with their own eyes - the basic fluorescence kinetic effect at first, in order to demonstrate them the basics of this technique and pique their interest for more complex methods and analyses. Therefore, we recommend start the laboratory practice with the demonstration of the 'Kautsky effect'.

For this purpose an intensive, e.g. LED, light source e.g. in the blue range is needed. Since the Chls are absorbing - of course with different efficiency - from the UV till ca. $700 \mathrm{~nm}^{4}$, other excitation wavelengths can be used as well. Any material for (partially) covering the leaf, and a red interference filter for detecting only the fluorescence wavebands and particularly excluding the excitation part of the spectrum, are needed. This filter can be used in form of a pair of red filter-spectacles and the students can observe the effect directly through these. The results described in this paper based on the demonstration kit developed by Opti-Sens Bt (e-mail: kocsanyi@eik.bme.hu). Or else, a capturing CCD camera can be equipped with a red filter and the images could be analyzed later on. As a result, a similar image sequence can be captured using a long (up to several seconds) exposition time which is showed on Figure 1. Perhaps, the two detections (one with human eye and other with CCD camera) can be realized simultaneously, if a bigger red-filter panel applied and students simultaneously with the CCD camera can observe the effect. We often use the leaves of Ficus benjamina L. or Laurus nobilis L. Necessarily we need a sample, which can be a living plant leaf or even an attached leaf, in order to simplify the setup. The experimental setup with a bigger red-filter panel shown on Figure 2 panel A, and the basic components of the setup using red filter-spectacles are shown on a photo on Figure 2 panel B. After this experiment with a living, functioning leaf, a fluorescence of a desiccated leaf need to be shown, which usually fluoresces at lower level and has almost no fluorescence kinetic.
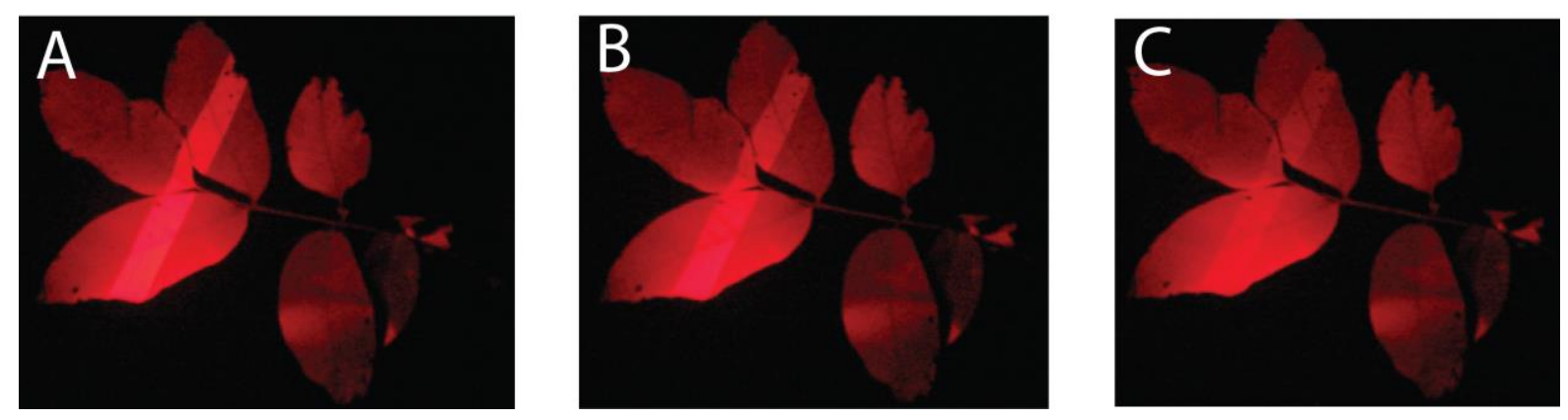

Figure 1. Captured images of the demonstration. The detected fluorescence image of partially dark adapted leaves of a Laurus nobilis L. plant A) immediately after finishing the dark adaptation and starting with the illumination B) after ca. 15 seconds illumination C) after ca. 90 seconds of illumination.

A

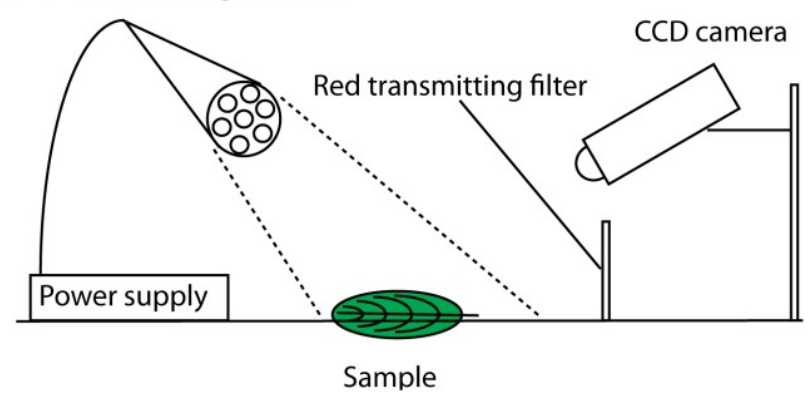

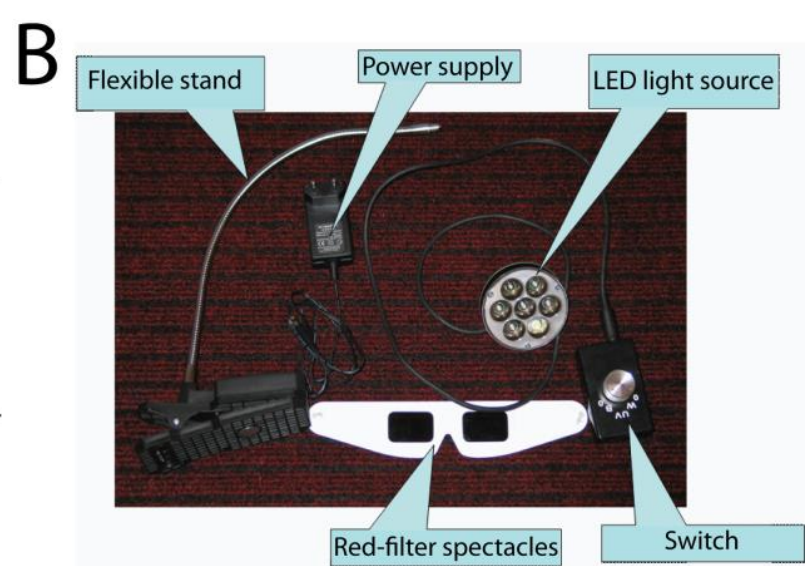

Figure 2. A) Experimental setup for the demonstration of the 'Kautsky effect' using a CCD camera. B) Basic components of the demonstration kit using red filter-spectacles. 


\section{MEASUREMENT OF FLUORESCENCE PARAMETERS USING THE INTELLIGENT FLUOROSENSOR}

After the students observed the basic process, we introduce them into more complex methods and teach them further biophysical phenomena. Usually, the fluorescence of leaves is monitored at dark adapted and light adapted states, and the photosynthesis is characterized by comparing the measured signals. The different adaptation states and the transition between them can be monitored by two different techniques: DC (continuous), total fluorescence measurement or PAM (pulse amplitude modulated), variable fluorescence signal. The total fluorescence emitted is always proportional to the excitation intensity which changes by several orders of magnitude. In case of the variable fluorescence, however, the measuring probe light, and the 'saturating' light and/or the so called actinic lights are separated. The last two determine the examinable light environment, whereas the measuring light provides the part of the excitation to which the response will be detected. As a result, only that portion of Chl- $a$ fluorescence which is excited by an extremely low dose (low intensity and duty factor or on/off ratio) pulsed light of constant intensity termed as the probe light is measured ${ }^{5}$.

The IFS provides total and variable fluorescence measurements simultaneously ${ }^{6}$. This has the advantage that the total fluorescence measurement - measured at two different wavebands at the two maxima of the Chl fluorescence - is based on the 'Kautsky effect', and the total fluorescence nicely shows the increasing photosynthetic activity and the simultaneously decreasing fluorescence signal. Therefore, these two fluorescence curves are easily understandable qualitatively after the demonstration experiment above. Since, in practice, we also apply so called saturating flashes, students see that the responses to the flashes also depend on the light adaptation of the photosynthetic reaction centers. From this, one can deduce the quenching analysis based on PAM technique with different (measuring, actinic and saturating) excitation light sources. Here we note that the final aim of the quenching analysis, based on the PAM technique, is to define the so-called photosynthetic energy partitioning, i.e. to define parameters (quantum yields) which will reflect the fraction of absorbed light energy utilized by particular processes (photochemistry vs. non-photochemical dissipations) $)^{7}$. Some important parameters are listed in Table 1.

Table 1. Some examples for parameters which can be calculated from the fluorescence induction kinetic measurements. More parameters are listed in several publications ${ }^{3,7}$. PSI and PSII denote the two photosystems of the functioning photosynthetic apparatus operating in series ${ }^{3}$.

\begin{tabular}{|c|c|}
\hline $\begin{array}{l}\text { Fluorescence } \\
\text { parameter }\end{array}$ & Physiological significance in case of PAM detection mode \\
\hline $\mathrm{F}_{0}$ & $\begin{array}{l}\text { Variable fluorescence response to measuring probe light only. Gives the minimal fluorescence from dark } \\
\text { adapted leaf. Level of fluorescence when PSII centers are open. }\end{array}$ \\
\hline $\mathrm{F}_{0}{ }^{\prime}$ & $\begin{array}{l}\text { Variable fluorescence response to measuring probe light and far-red light (that selectively excites PSI). } \\
\text { Gives the minimal fluorescence from light adapted leaf. Level of fluorescence when PSII centers are open. }\end{array}$ \\
\hline $\mathrm{F}_{\mathrm{m}}$ & $\begin{array}{l}\text { Variable fluorescence response to measuring probe light and saturating flashes. Gives the maximal } \\
\text { fluorescence from dark adapted leaf. Level of fluorescence when PSII centers are closed. }\end{array}$ \\
\hline $\mathrm{F}_{\mathrm{m}}{ }^{\prime}$ & $\begin{array}{l}\text { Variable fluorescence response to measuring probe light, actinic light and saturating flashes. Maximal } \\
\text { fluorescence from light adapted leaf. Level of fluorescence when PSII centers are closed. }\end{array}$ \\
\hline $\mathrm{F}_{\mathrm{v}}=\mathrm{F}_{\mathrm{m}}-\mathrm{F}_{0}$ & $\begin{array}{l}\text { Variable fluorescence from dark adapted leaf. Demonstrates the ability of PSII to perform primary } \\
\text { photochemistry. }\end{array}$ \\
\hline $\mathrm{F}_{\mathrm{v}}^{\prime}=\mathrm{F}_{\mathrm{m}}{ }^{\prime}-\mathrm{F}_{0}{ }^{\prime}$ & $\begin{array}{l}\text { Variable fluorescence from light adapted leaf. Demonstrates the ability of PSII to perform primary } \\
\text { photochemistry. }\end{array}$ \\
\hline $\mathrm{F}_{\mathrm{q}}^{\prime}=\mathrm{F}_{\mathrm{m}}{ }^{\prime}-\mathrm{F}^{\prime}$ & $\begin{array}{l}\text { Difference in fluorescence from Fm' and the actual fluorescence without flash. Photochemical quenching of } \\
\text { fluorescence due to open PSII centers. }\end{array}$ \\
\hline $\mathrm{F}_{\mathrm{v}} / \mathrm{F}_{\mathrm{m}}$ & Maximum quantum efficiency of PSII photochemistry from a dark adapted leaf. \\
\hline $\mathrm{F}_{\mathrm{v}}^{\prime} / \mathrm{F}_{\mathrm{m}}^{\prime}$ & hemistry at a given light int \\
\hline
\end{tabular}

The measurement protocol of the IFS is completed by the leaf surface temperature measurement ${ }^{6,8}$. The core of the measurement protocol is shown in Figure 3 in case of a living leaf with functioning photosynthetic apparatus. The 
detailed measurement protocol is described by Barócsi ${ }^{6}$. Additionally, the fluorescence of a fully desiccated leaf should also be presented, which is only changing stepwise between different levels indicating that no more biophysical processes exist in a fully desiccated leaf.

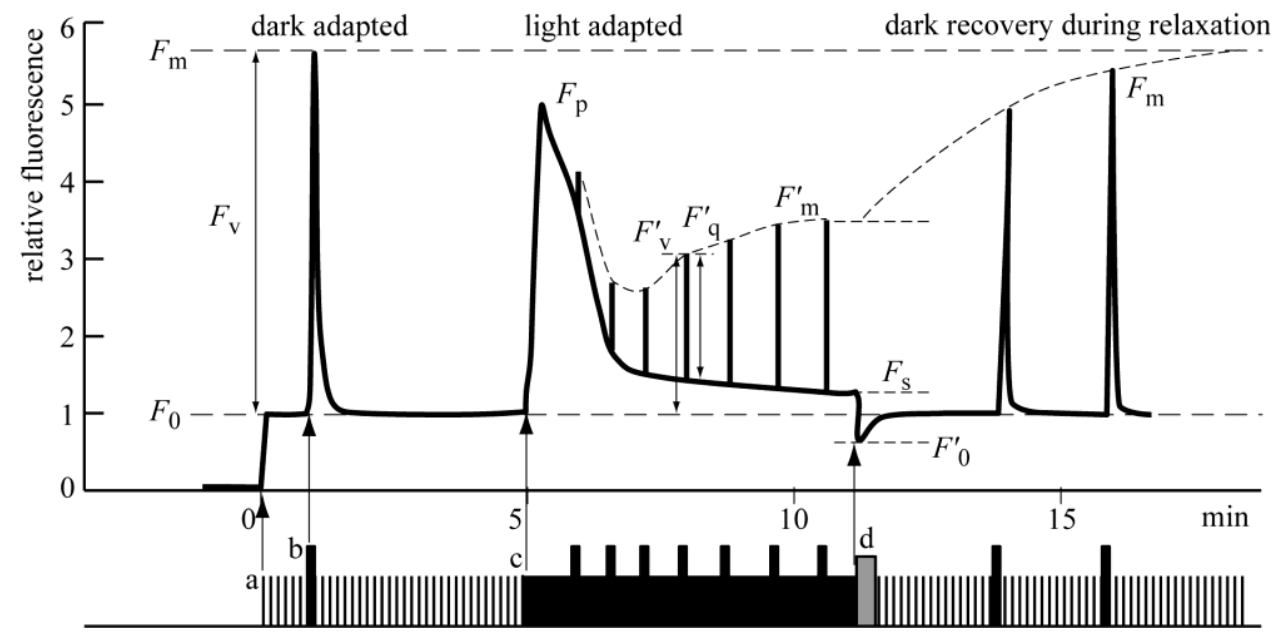

Figure 3. The core of the measurement protocol of the IFS. Letters mark the different excitations: (a) measuring probe light, (b): saturating flas, (c) actinic light and (d) far-red light

The IFS instrumentation is described in detail by Barócsi ${ }^{6}$, and the concept of the instrumentation is shown in Figure 4. The IFS tool is also capable to record the excitation kinetics ${ }^{9-10}$. This kind of measurement is based on a feedback mechanism. When keeping the fluorescence constant by the feedback mechanism of the IFS, the change of fluorescence yield during the dark-to-light transfer is compensated by an inverse change of the excitation light. The feedback mechanism reacts to the fluorescence changes of the sample and adjusts the laser power individually to the sample. Another feedback regulation adjusting the intensity of the excitation light in order to keep the Chl fluorescence stable is used by an instrument with a dual wavelength excitation (DUALEX) ${ }^{11}$. This technique is developed to acquire rapid information about the absorption characteristic of the leaf tissue and thus is used to 'fully eliminate any artifacts caused by variable Chl fluorescence' as has been demonstrated for quality assessment in grape vine production ${ }^{12}$. Therefore, it is also important to show this kind of measurement protocol for students, which has a clear potential in precision agriculture. Furthermore, the implementation of such a measurement technique is interesting for students studying physics or engineering.
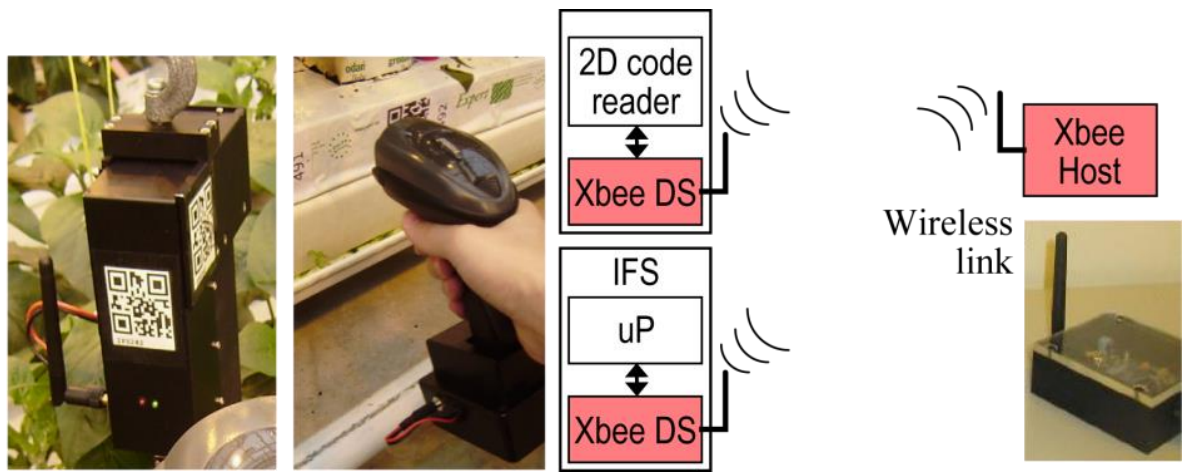

Figure 4. The concept of the IFS instrumentation 


\section{CONCLUSIONS}

The fluorescence induction kinetics of leaves is a complex signal, which depends on the concentration of the fluorophore Chl- $a$, on the leaf structure, on the photosynthetic activity and on the leaf's optical properties. Here, we propose a progressive introduction of students - with the presumption having no preliminary experience - into this multidisciplinary research field. We start with the demonstration of the 'Kautsky effect', which shows the varying fluorescence caused by the change of fluorescence yield to photosynthetic processes during the dark-to-light transfer. Afterwards, more complex fluorescence studies are applied using IFS. The PAM measurement protocol allows the positioning of the photosynthetic energy using quenching analysis. Finally, the measurement protocol with feedback regulation is demonstrated. The proposed laboratory practice can be a good starting point for students interested for this multidisciplinary research field.

\section{ACKNOWLEDGEMENTS}

The project was partially funded by „TÁMOP-4.1.1.C-12/1/KONV-2012-0005 - Preparation of the concerned sectors for educational and R\&D activities related to the Hungarian ELI project" supported by the European Union and cofinanced by the European Social Fund.

\section{REFERENCES}

[1] Kautsky, H. and Hirsch, A., "Neue Versuche zur Kohlenstoffassimilation," Naturwissenschaften 19, 964 (1931).

[2] Buschmann, C., "Variability and application of the chlorophyll fluorescence emission ratio red/far-red of leaves," Photosynth Res. 92, 261-271 (2007).

[3] Papageorgiou, G.C., Govindjee, [Chlorophyll a Fluorescence, a Signature of Photosynthesis], Springer, Dordrecht (2004).

[4] Buschmann C., Lenk, S., Lichtenthaler, H. K., "Reflectance spectra and images of green leaves with different tissue structure and chlorophyll content," Israel Journal of Plant Sciences 60(1-2), 49-64 (2012).

[5] Hout, Y., Babin M., "Overview of fluorescence protocols: theory, basic concepts and practice," in: D. J. Suggett et al. (eds.) Chlorophyll a Fluorescence in Aquatic Sciences: Methods and Applications, Developments in Applied Phycology. Springer 31-74 (2011).

[6] Barócsi, A., "Intelligent, net or wireless enabled fluorosensors for mass monitoring of assorted crops," Measurement Science and Technology, 24(2), 1-14 (2013).

[7] Lazár D., " Parameters of photosynthetic energy partitioning," Journal of Plant Physiology 175 131-147 (2015).

[8] Solti, Á., Lenk, S., Mihailova, G., Mayer, P., Barócsi, A., Georgieva, K., "Effects of habitat light conditions on the excitation quenching pathways in desiccating Haberlea rhodopensis leaves: An Intelligent FluoroSensor study," Journal of Photochemistry and Photobiology B: Biology 130 217-225 (2014).

[9] Barocsi, A., Lenk, S., Kocsányi, L., Buschmann, C., "Excitation kinetics during induction of chlorophyll a fluorescence," Photosynthetica 47(1), 104-111 (2009).

[10] Buschmann, C., Konanz, S., Zhou, M., Lenk, S., Kocsányi, L., Barócsi, A., "Excitation kinetics of chlorophyll fluorescence during light-induced greening and establishment of photosynthetic activity of barley seedlings," Photosynthetica 51(2), 221-230 (2013).

[11] Goulas, Y., Cerovic, Z.G., Cartelat, A., Moya, I., "Dualex: a new instrument for field measurements of epidermal ultraviolet absorbance by chlorophyll fluorescence," Applied Optics 43, 4488-4496 (2004).

[12] Cerovic , Z.G., Moise, N., Agati, G., Latouche, G., Ben Ghozlen, N., Meyer, S., "New portable optical sensors for the assessment of winegrape phenolic maturity based on berry fluorescence," Journal of Food Composition and Analysis 21, 650-654 (2008). 